\title{
Authoring and Dynamic Generation of Adaptive E-courses
}

\author{
Rosa M. Carro ${ }^{1}$, Manuel Freire ${ }^{1}$, Estefanía Martín ${ }^{1}$, Alvaro Ortigosa ${ }^{1}$, \\ Pedro Paredes ${ }^{1}$, Pilar Rodríguez ${ }^{1}$, and Johann Schlichter ${ }^{2}$ \\ ${ }^{1}$ Escuela Politécnica Superior, Universidad Autónoma de Madrid \\ 28049 Madrid, Spain \\ \{Rosa.Carro, Manuel.Freire, Estefania.Martin, Alvaro.Ortigosa, \\ Pedro.Paredes, Pilar.Rodriguez\}@ii.uam.es \\ ${ }^{2}$ Institute for Informatics, Technical University of Munich \\ 85748 Munich, Germany \\ Schlichter@in.tum.de
}

\begin{abstract}
Adaptive hypermedia constitutes a pretty rich resource for developing web-based courses. With the aim of dynamically generating adaptive ecourses, we have developed the TANGOW system which, starting from the course components and their adaptation capabilities (specified independently and out of the adaptation engine), generates different courses for students with different profiles, supporting several adaptation strategies. An integral part of any adaptive hypermedia system is the set of authoring tools to specify the course components and their adaptation capabilities. Without adequate tool support, authors may feel that it is "not worth the effort" to add adaptation to their courses. However, the development of this type of tools is not an easy task. The main goal of our authoring and visualization tools is to provide a simple interface to create such courses. This demo would demonstrate i) the dynamic generation of tailored e-courses that include individual and collaborative activities and ii) the use of authoring tools for the creation of such courses.
\end{abstract}

\section{The Delivery System}

The current version of TANGOW [1][4] supports the adaptation, for each student, of:

- The presence/absence of topics and activities.

- The organization of topics, which leads to the course structure.

- The navigational guidance provided, which can be different through the course.

- The requirements to access a topic or to start accomplishing an activity, which can be different for different types of students.

- The multimedia contents used to generate the pages presented to the students. The most suitable version can be selected for each student at runtime.

- The collaboration workspaces to support collaboration activities among users, including the specific problem to be proposed to each group and the set of tools to support the collaboration, which can be chosen depending on the users' preferences and learning styles, among other criteria. 
Concerning the users' aspects taken into account during the adaptation process, the system can use any information considered as relevant by the course designer, who can specify it at the beginning of the course creation. Some aspects considered are:

- Personal features, such as age and language.

- Learning style: visual/textual, sequential/global, sensitive/intuitive, etc [3].

- Preferences: type of information desired (specific/general) or strategy (theoryexamples-exercises, exercises-examples-theory), among others.

- Actions performed and scores got during the interaction with the course.

The way courses are generated at runtime according to the user profile and to the adaptation possibilities of each course[4] will be shown in the demonstration.

\section{The Authoring Tools}

In order to support the specification of the adaptation possibilities of a TANGOWbased course, we created a formalism based on the use of teaching tasks and teaching rules, which has been recently extended to support adaptation of collaboration activities [1]. We have developed authoring tools to help the course designers to specify each course and its adaptation possibilities [4].

Course builder tool. It covers the entire authoring process, including course design, creation and edition of course components (rules, tasks and hypermedia fragments) and the adaptation capabilities. It supports the simulation of the course for different student profiles, and course overview generation.

Graph-based course builder tool. It supports the generation and exploration of highlevel course overviews. Courses are graph-based represented and can be visualized in different ways by using filtering and clustering utilities. This tool also allows the edition of the course components [2].

Cooperative workspace tool. It will facilitate the definition of the adaptive cooperative workspaces included in the latest version of the system.

\section{References}

1. Carro, R.M., Ortigosa, A., Martín, E., Schlichter, J.: Dynamic Generation of Adaptive Web-based Collaborative Courses. In: Decouchant, D. and Favela, J. (eds.): Groupware: Design, Implementation and Use. LNCS 2806. Springer-Verlag Berlin Heidelberg (2003) 191-198

2. Freire, M., Rodriguez, P.: A graph-based interface to complex hypermedia structure visualization. In: Proceedings of Advanced Visual Interfaces 2004. ACM-Press (2004) In Press

3. Paredes, P., Rodriguez, P.: Considering Sensing-Intuitive Dimension to ExpositionExemplification in Adaptive Sequencing. In: De Bra, P., Brusilovsky, P. and Conejo, R. (eds.): Adaptive Hypermedia and Adaptive Web-Based Systems. LNCS 2347. SpringerVerlag, Berlin Heidelberg (2002) 556-559

4. TANGOW and related tools: http://tangow.ii.uam.es/opah 